DOI:

10.1038/nrc2140

URLs

EGFR

http://www.ncbi.nlm.nih.gov/ entrez/query.fcgi?db=gene\&c $\mathrm{md}=$ Retrieve\&dopt=full report\&list_uids $=1956$

EREG

http://www.ncbi.nlm.nih.gov/ entrez/query.fcgi?db=gene\&c $\mathrm{md}=$ Retrieve\&dopt=full reportElist_uids $=2069$

\section{$\operatorname{cox} 2$}

http://www.ncbi.nlm.nih.gov/ entrez/query.fcgi?db=gene\&c $\mathrm{md}=$ Retrieve\&dopt $=$ Graphics $\mathcal{E}$ list_uids $=5743$

MMP1

http://www.ncbi.nlm.nih.gov/ entrez/query.fcgi?db=gene\&c $\mathrm{md}=$ Retrieve\&dopt=full report\&list_uids $=4312$

MMP2

http://www.ncbi.nlm.nih.gov/ entrez/query.fcgi?db=gene\&c $\mathrm{md}=$ Retrieve\&dopt=full reportElist_uids=4313

\section{Breast cancer}

http://www.cancer.gov/ cancertopics/types/breast

METASTASIS

\title{
To grow and spread
}

Joan Massagué and colleagues have previously identified a lung metastasis gene-expression signature (LMS) in human breast cancer cells, of which a subset of genes supports both primary tumour growth and metastasis, whereas others support just metastasis. How do these gene subsets differ in their function?

The epidermal growth factor receptor (EGFR) and one of its ligands, epiregulin (EREG), cyclooxygenase 2 (COX2) and the matrix metalloproteinases MMP1 and MMP2 were independently shown to be downstream mediators of vascular endothelial growth factor. These genes are also part of the LMS, so Massagué and colleagues asked whether they enable vascular remodelling in developing tumours and if this is relevant to metastasis.

Reducing the individual expression of EREG, COX2, MMP1 and $M M P 2$ using short hairpin RNA interference in a subpopulation of a human breast cancer cell line that has lung metastatic capacity (LM2 cells) had little effect on orthotopic tumour cell growth in nude mice, but the silencing of all four genes severely diminished growth. The combined knockdown of three other LMS genes, IL13RA2, SPARC and VCAM1, had little effect on tumour growth, but did limit the extent of lung metastasis, confirming the existence of metastasis genes that affect primary tumour growth and those that do not. Examination of the tumours formed by LM2 cells in which EREG, COX2, MMP1 and $M M P 2$ are silenced showed reduced branching of the vasculature and attenuated vascular permeability, indicating that these genes are responsible for the dilated, tortuous and leaky vasculature found in aggressive tumours. But what about metastasis? Knockdown of EREG, COX2, MMP1 and MMP2 significantly reduced the formation of lung metastases from the tumours as well as after tail vein injection, most probably owing to a block in extravasation.

The combined use of pan-MMP (GM6001), COX2 (celecoxib) and EGFR (cetuximab) inhibitors also reduced primary tumour growth in an orthotopic human breast cancer metastasis mouse model, owing to vascular defects. They also reduced levels of circulating tumour cells from the primary tumour and prevented extravasation of these cells in the lungs. Interestingly, the tumour cells in the lungs survived within the vasculature, and removal of the inhibitors after 4 weeks enabled the growth and invasion of these cells into the lungs. Notably, the

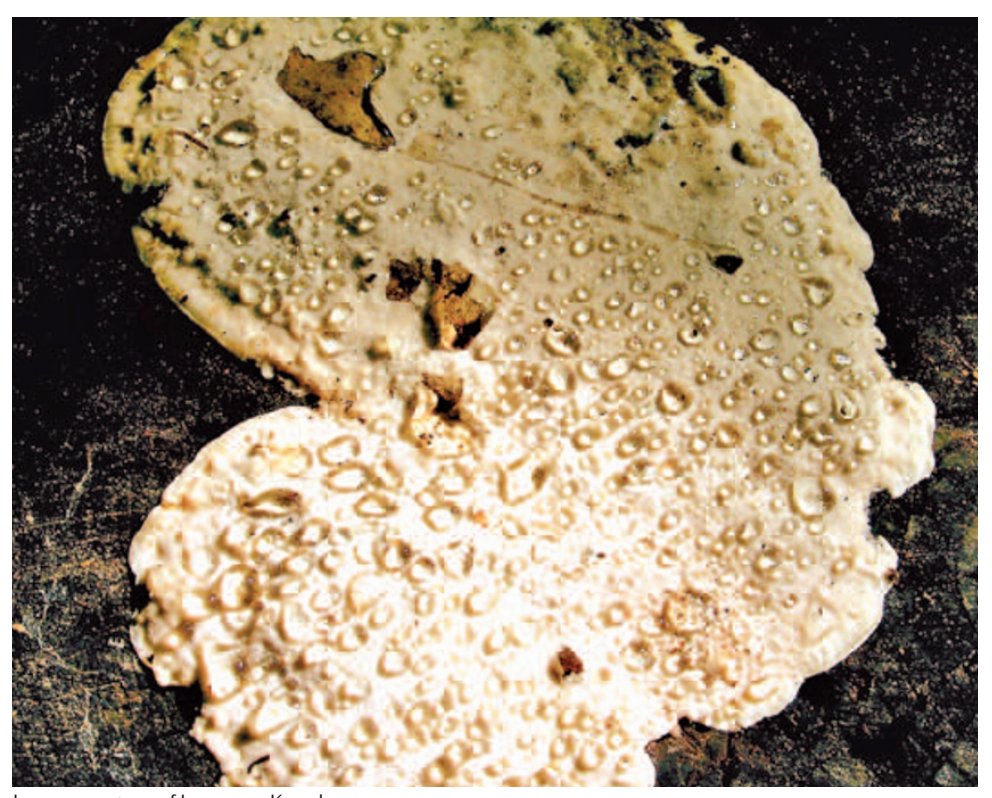

Image courtesy of Laurence Keogh addition of GM6001 did not increase the inhibitory effects of celecoxib and cetuximab, and importantly, celecoxib and cetuximab blocked the growth and metastasis of tail-vein-injected cells isolated from breast cancer patients with lung metastases.

These data indicate that specific genes both facilitate primary tumour growth and metastasis, which the authors term 'metastasis progression genes', and are distinct from 'metastasis virulence genes' that have no function in primary tumour growth. Moreover, identifying the biological role of these genes has indicated that inhibitors of COX2 and EGFR might be useful as anti-metastatic treatments for breast cancer.

Nicola McCarthy

ORIGINAL RESEARCH PAPER Gupta, G.P. et $a$. Mediators of vascular remodelling co-opted for sequential steps in lung metastasis. Nature $\mathbf{4 4 6}$, 765-770 (2007) 